Mariendistel bewährt bei der Therapie von Lebererkrankungen

\section{Neue Ansätze auch bei Hepatitis C}

Silymarin - der Extrakt aus der Mariendistel hat sich bereits bei vielen hepatologischen Indikationen als sehr wirkungsvoll erwiesen. Neuere Studiendaten deuten darauf hin, dass Silibinin, die aktive Komponente aus Silymarin, ein wirksames antivirales Agens gegen Hepatitis C sein kann. Dies gilt besonders für Patienten, die auf eine Standardtherapie nicht ansprechen.

Mit dem patentierten Originalwirkstoff in Lega$\operatorname{lon}^{\circledR}$ (mit der aktiven Verbindung Silibinin) verfügt der Hersteller Madaus über ein in der Lebertherapie sehr bewährtes Arzneimittel mit einer viel versprechenden Zukunftsperspektive.

Aktuelle Lebertherapie - Komplementärmedizinische Erweiterung

Obwohl die Sterberate bei chronischen Lebererkrankungen in den letzten Jahren zurückgegangen ist, sind die Möglichkeiten einer spezifischen Therapie bei Hepatitis B und C weiterhin begrenzt. Die Folge: Eine Vielzahl der Patienten, die auf die Standardtherapie nicht ansprechen, setzen auf alternative Behandlungsmethoden. Der Einsatz von Silymarin-Präparaten stellt eine sinnvolle Option dar, erläutert Privat-Dozent Dr. med. Franz-Josef Vonnahme, Hameln. Die positiven Eigenschaften von Silymarin mit der aktiven Verbindung Silibinin sind seine mannigfaltigen hepatoprotektiven und antiinflammatorischen Effekte. Besonders therapeutisch interessant ist jedoch der antifibrotische Effekt von Silymarin, so Vonnahme. In einer klinischen Langzeitstudie konnte bereits eine deutliche Verlängerung der Lebenszeit bei Patienten mit einer Leberzirrhose belegt werden. Insgesamt gesehen bezeichnet Vonnahme Silymarin als «hoch interessantes Medikament» bei der Behandlung chronischer Lebererkrankungen, das in der komplementären Medizin weltweit auf Grund seiner Wirksamkeit und guten Verträglichkeit Verwendung findet.

Silymarin - Therapeutikum mit antifibrotischer Wirkung

Professor Detlef Schuppan aus Boston sieht in Silymarin ebenfalls einen Wirkstoff, der sich hervorragend zur Therapie bei Patienten mit einer Leberfibrose/Zirrhose eignet, die auf eine kausale Therapie nicht ansprechen oder bereits eine fortgeschrittene Fibrose/Zirrhose haben und deshalb eine antifibrotische Therapie benötigen. Zurzeit ist Silymarin das einzige als Lebertherapeutikum zugelassene Präparat, für das experimentell eine klare antifibrotische Wirkung belegt werden konnte. Gegenwärtig wird der hepatoprotektive und antifibrotische Effekt von Legalon ${ }^{\circledR}$ in NIH-geförderten klinischen Studien an Patienten mit chronischer Hepatitis $\mathrm{C}$ und nichtalkoholischer Steatohepatitis untersucht.

\section{Quelle}

Pressegespräch «Mariendistel bewährt bei der Therapie von Lebererkrankungen. Neue Ansätze auch bei Hepatitis C» am 2. Oktober 2008 in Berlin.

Weitere Informationen bei

Adlexis GmbH

Karin Bretz

Liebherrstraße 10, 80538 München

Tel.+49 89 2020816-0, Fax -10

karin.bretz@adlexis.com

\section{Promotion in der Komplementär- medizin}

Die Karl und Veronica Carstens-Stiftung veranstaltet am 6. und 7. März 2009 ihr jährliches Promotionsseminar. Es richtet sich an Studierende der Medizin, die in ihrer Doktorarbeit ein Thema aus der Naturheilkunde oder Homöopathie bearbeiten wollen. Bewerbungsschluss für das Seminar ist der 15. Januar 2009.

An den zwei Seminartagen wird gezeigt, wie eine wissenschaftliche Arbeit richtig zu planen, durchzuführen und natürlich auch erfolgreich zum Abschluss zu bringen ist. Studierende, die auf der Suche nach einem
Thema für ihre Doktorarbeit sind oder die bereits eine Promotionsidee aus dem Bereich Komplementärmedizin haben, können sich bewerben. In einem anschließenden Workshop wird es die Gelegenheit geben, gemeinsam Ideen für die Promotion zu entwickeln sowie die eigenen Vorstellungen zu diskutieren und zu konkretisieren. Am Ende des Seminars werden exemplarisch einige Promotionsarbeiten vorgestellt. Im Rahmen des Promotionsförderprogramms werden von der Carstens-Stiftung für einzelne Doktorarbeiten Stipendien vergeben.

Veranstaltungsort ist die Carstens-Stiftung in Essen. Aufenthalt, Übernachtung und Verpflegung werden erstattet. Die Reisekosten müssen von den Teilnehmern selbst getragen werden.

Bewerbungen mit kurzer schriftlicher Begründung des Interesses sowie ein tabellarischer Lebenslauf sind an die Carstens-Stiftung zu richten.

Bewerbung und weitere Informationen bei Carstens-Stiftung

Am Deimelsberg 36, 45276 Essen

Tel.+49 201 56305-34, Fax -30

www.carstens-stiftung.de

\title{
Ticker+++ Ticker+++ Ticker+++ Ticker+++ Ticker+++ Ticker+++ Ticker+++
}

Orthotec s.l. kieferorthopädische Geräte Wiesbaden. Die Vermessung des Kiefers ist für die Planung jeder kieferorthopädischen Behandlung unverzichtbar. Bisher war dies nur durch Fernröntgenseitenaufnahmen möglich. Seit kurzem gibt es eine strahlungsfreie Alternative: noXrayCeph ${ }^{\circledR}$ wurde von dem Wiesbadener Kieferorthopäden Dr. med. dent. Thorsten Brandt in Zusammenarbeit mit der Poliklinik für Kieferorthopädie, Universität Frankfurt, entwickelt.

Weitere Informationen bei

Dr. rer. nat. Bettina Jung

Konrad-Adenauer-Ring 27, 65187 Wiesbaden e-mail@ bettinajung.de
Repha GmbH. Weltweit sterben jedes Jahr mehr Menschen infolge antibiotika-resistenter Keime als an Aids. Daher plädieren immer mehr Experten dafür, Antibiotika gezielter einzusetzen und zum Beispiel bei unkomplizierten viralen und bakteriellen Infektionen auf pflanzliche Antibiotika wie Angocin ${ }^{\circledR}$ Anti-Infekt N auszuweichen.

Die Patientenbroschüre «Pflanzliche Antibiotika - die sanfte Heilung aus der Natur» können Ärzte bestellen unter:

Repha GmbH

Alt-Godshorn 87, 30855 Langenhagen www.repha.de
SELF e.V., die europäische Liga für ein sicheres Patienten-Therapie-Selbstmanagement, stiftet den «SELF-Europa-Award 2009», der von einem unabhängigen Expertengremium aus Klinik, Praxis und Forschung zuerkannt wird. Mit dem Award soll ein für die Selbstmedikation geeignetes Arzneimittel gewürdigt werden. Bewerbungen können bis zum 21. November 2008 eingereicht werden. Die Angaben zu den Kriterien sowie weitere Informationen bei:

SELF e.V.

Geschäftsstelle

Exerzierplatz 30, $24103 \mathrm{Kie}$ www.self-global.de 